\title{
Computation Programming of McCabe-Thiele and Ponchon-Savarit Methods for SHORT-CUT Distillation Design
}

\author{
Chavdar Chilev ${ }^{1,2, *}$, Moussa Dicko ${ }^{2}$, Patrick Langlois ${ }^{2}$, Farida Lamari ${ }^{2}$ \\ ${ }^{1}$ Chemical Engineering, University of Chemical Technology and Metallurgy, Sofia, Bulgaria \\ ${ }^{2}$ Chemical Engineering, CNRS LSPM University Sorbonne Paris Nord, Villetaneuse, France
}

Email address:

chavdar_chilev@uctm.edu (C. Chilev)

${ }^{*}$ Corresponding author

To cite this article:

Chavdar Chilev, Moussa Dicko, Patrick Langlois, Farida Lamari. Computation Programming of McCabe-Thiele and Ponchon-Savarit Methods for SHORT-CUT Distillation Design. International Journal of Science, Technology and Society. Vol. 9, No. 6, 2021 , pp. $263-274$. doi: $10.11648 /$ j.ijsts.20210906.12

Received: October 3, 2021; Accepted: October 25, 2021; Published: November 5, 2021

\begin{abstract}
Using modern computer programming resources, a computer code has been developed in the MatLAB programming environment, which allows the use of the McCabe-Thiele and Ponchon-Savarit methods for SHORT-CUT distillation design. The McCabe-Thiele and Ponchon-Savarit methods are easy to apply, are not time consuming, and allow the easy visualization of the interrelationships among variables. In order to describe all the programming steps of these methods, a combination of different types of MatLAB functions has been used. The optimum reflux ratio is determined by using volume criteria, whichallows minimizing the volume of the distillation column and thereby reducing the total cost of a distillation unit. To evaluate the accuracy of the results, a comparison between the results produced by graphical methods and those calculated by other SHORT-CUT methods and rigorous calculations has been carried out. To perform this, the ChemCAD 7.1.5 simulator has been used. The SHORT-CUT distillation module in this simulator uses the Fenske-Underwood-Gilliland (FUG) method. For rigorous estimation, the SCDS multi-stage vapor-liquid equilibrium module in ChemCAD software environment has been used. SCDS is a rigorous multi-stage vapor-liquid equilibrium module which simulates any single column calculation including distillation columns, absorbers, reboiler and strippers. The results produced by graphical methods are closer to the rigorous-calculation results than to the FUG SHORT-CUT method ones, with respect both to the reflux ratio and to the bottom and top light-key mass fraction.
\end{abstract}

Keywords: Distillation, Short-Cut Methods, Computer Simulations, McCabe-Thiele, Ponchon-Savarit

\section{Introduction}

Before the advent of the modern digital computer, various "SHORT-CUT" methods had been developed to simplify the task of designing multicomponent distillation columns. Though computer programs would normally be available for the rigorous-solution equations, SHORT-CUT methods are still useful in the preliminary design work, and as an aid in defining problems for computer solution. Intelligent use of the SHORT-CUT methods can indeed reduce both computer time and costs.

The SHORT-CUT methods available for distillation design processes can be divided into two classes: empirical methods and simplified methods [1-3]. The first ones are based on the performance of operating columns and on results of rigorous designs. Typical examples of these methods are the Gilliland's [4] and the Erbar-Maddox correlations [5]. The other methods are based on the simplification of the rigorous stage-by-stage procedures in order to enable the calculations to be done by using hand calculators or graphically. Typical examples of this approach are the McCabe-Thiele [6] and the Ponchon-Savarit methods [7, 8].

The first class of methods has been implanted in modern simulation software such as ASPEN ONE, ChemCAD, or ProSIM while the McCabe-Thiele and Ponchon-Savarit methods are two graphical methods used for the design of 
multistage distillation columns for binary systems. They are very useful for didactical purposes and for preliminary calculations. The basic advantages of these methods are the easy visualization of the interrelationships among variables [9] and the use of clearly defined criteria for calculating the optimal reflux ratio in the distillation columns (see part 2.4). The disadvantages relate to their effort- and time-consuming aspects, which are due to the graphical constructions of these hand-executed methods. This is the reason why they are not included in the package methods of modern computer simulator for chemical engineering processes.

The purpose of this study is to use modern computer programming resources to develop a computer code in the MatLAB programming environment that allows the use of graphical methods for SHORT-CUT distillation design which can be integrated into commercial chemical engineering software products through the Computer Aided Process Engineering Open (CAPE OPEN) interface standard.

For a better visualization when presenting the developed program codes, the results of calculations for a selected real system composed of acetone/n-butanol have been displayed.

\section{Model Development}

The graphical McCabe-Thiele and Ponchon-Savarit methods are well known in the literature [10-13]. Therefore, a detailed thermodynamic description of these methods is not hereby provided. It is directly described how the individual details (steps) of the two methods are realized according to the computer code.

\subsection{Thermodynamic Calculations}

Regardless of which method is used for the design of distillation columns, the first step always consists in determining the thermodynamic parameters of the selected system. When using the McCabe-Thiele and Ponchon-Savarit methods, the thermodynamic parameters are the liquid-vapor equilibrium and the enthalpies of the liquid and vapor phases. There are two approaches to determine these parameters.

The first approach involves the use of a commercial simulator such as ASPEN ONE or ChemCAD. In this case, the code created in MatLAB should be connected to the simulator using the CAPE OPEN interface standard. Once the connection between the MatLAB code and the simulator is carried out, any feature integrated in the simulator models to determine the necessary thermodynamic parameters can be used. This is a good approach for calculating the thermodynamics of the selected system, because rigorous models are integrated into the simulators to calculate the required parameters and there is a possibility to combine different models. Whichever thermodynamic model from the simulator library is to be selected (except for the UNIFAC activity coefficient model), it will however require the availability of experimental vapor-liquid equilibrium data [14]. The disadvantage of this approach is that MatLAB code cannot work alone and a commercial simulator must be available.

The second approach relates to the possibility of directly determining the thermodynamic parameters from experimental vapor-liquid equilibrium data only. On the basis of such data, the vapor-liquid equilibrium is approximated and the enthalpies of the liquid and vapor phases are calculated. The approximation is performed using the "spline" function in MatLAB, which performs an approximation of the experimental data. The syntax of this function is: $Z=\operatorname{spline}(X, Y)$. This function returns the piecewise polynomial form of the cubic spline interpolant. The obtained result in MatLAB is in the form of an information array and does not represent a specific mathematical expression. To convert and use the result of the information array in order to calculate the required parameters, the "ppval" function is used. It has the following syntax: $V=\operatorname{ppval}\left(Z, X_{1}\right)$. This function returns the value of the piecewise polynomial $f$ contained in $Z$, at the entries of $X_{1}$. Thus, a combination of the two functions spline and ppval, experimental vapor-liquid equilibrium data and enthalpy data for both phases are approximated. The use of a spline function allows for the approximation of highly nonlinear and discontinuous functions, and it allows for the statistical aggregation of disperse experimental points. This gives good accuracy in the approximation of the experimental data.

The created MatLAB code allows to select one of the two approaches discussed above, i.e. either using the CAPE OPEN interface standard with a commercial simulator or the direct approximation of experimental vapor-liquid equilibrium data. The results presented in this work relate to the latter option.

\subsection{McCabe-Thiele Method}

The McCabe-Thiele and Ponchon-Savarit methods have been developed for binary systems. In order to apply these methods to multicomponent systems, the concept of "key" components must be defined. Key components are the two components of a multicomponent mixture between which separation is desired. The light key $(L K)$ refers to the component that is desired to keep out of the bottom productand shall accumulate in the top product. The heavy key $(H K)$ reversely refers to the component that is desired to keep out of the top product and shall accumulate in the bottom product. The keys can therefore be treated as a pseudo-binary pair and the McCabe-Thiele and PonchonSavarit methods can be applied to this pseudo-binary system for the separation of multicomponent systems.

The description of the development of graphical design procedures starts with the vapor-liquid equilibrium. As mentioned in the previous part (2.1), the approximation has been performed by a spline function. For the acetone/n-butanol system, the acetone is $L K$ and experimental data on vapor equilibrium have been taken from the literature [15]. In Figure 1, a comparison between experimental and model results is presented (red curve). The $x$-axis shows the molar concentrations of $L K$ in the liquid phase and the $y$-axis shows the molar concentrations of $L K$ in the vapor phase. The figure shows a good agreement between model and experimental values. 


\subsubsection{Construction of the Operating Lines of the McCabe-Tile Method}

This methods hinges upon the fact that, as an approximation, the operating lines on $x-y$ diagram can be considered straight for each section of the fractionator $[6,11$, $12,16]$. The parameters available are the concentration of $L K$ in the top product (in the condenser) $-x_{D}$, the concentration of $L K$ in the bottom product (in the reboiler) $-x_{W}$, and the concentration of $L K$ in the feed stream $-x_{f}$. For the acetone/n-butanol system, the following starting values for these parameters have been selected: $x_{f}=0.56, x_{D}=0.95$, and $x_{W}=0.03$.

$$
\begin{gathered}
y=\frac{R}{(R+1)} x+\frac{x_{D}}{(R+1)} \\
y=\frac{f+R}{(R+1)} x-\frac{f-1}{(R+1)} x_{W}
\end{gathered}
$$

Equations (1) and (2) represent the rectifying operating line (ROL) and the stripping operating line (SOL) respectively. In equations (1) and (2), the parameter $R$ corresponds to the reflux ratio of the column. In order to be able to calculate the number of theoretical degrees by the two graphical methods, the parameter $R$ must be assessed. In order to determine an optimum reflux ratio $\left(R_{\text {opt }}\right)$, the minimum reflux ratio $R_{\min }$ must first be determined.
Calculations of the optimum reflux ratio $\left(R_{\text {opt }}\right)$ are given in more detail in Part 2.4. Assuming that this parameter for the acetone/n-butanol system and for the McCabe-Thiele method is $R_{\text {opt }}=0.594$, equation (1) provides:

$$
y=\frac{0.594407}{(0.594407+1)} x+\frac{0.95}{(0.594407+1)}=0.3728 x+0.5958
$$

The feed entering the distillation column may consist of liquid, vapor or a mixture of both. Some portions of the feed go as the liquid and vapor stream to the rectifying and stripping sections. This is represented by the parameter $f$ in equation (2).

In order to construct the operating lines, it is necessary to find (calculate the coordinates) the end points bounding these lines and corresponding to certain points through the height of the column. The first point of ROL corresponds to the condenser and the end point of SOL corresponds to the reboiler. One consideration of the McCabe-Tile method consists in the equality of concentrations in the liquid and vapor phases in both the condenser and the reboiler: $y_{W}=x_{W}$ and $y_{D}=x_{D}$. Thus, the coordinates of the first point of the ROL (to the top) $\operatorname{are}\left(x_{D}, x_{D}\right)$. Similarly, those of the end point of the SOL are $\left(x_{W}, x_{W}\right)$ These two points lie on the diagonal of the diagram. For the acetone/n-butanol system, the corresponding points are: $B(0.95,0.95)$ - for the ROL and $C(0.03,0.03)$ - for the SOL respectively, as shown in Figure 1.

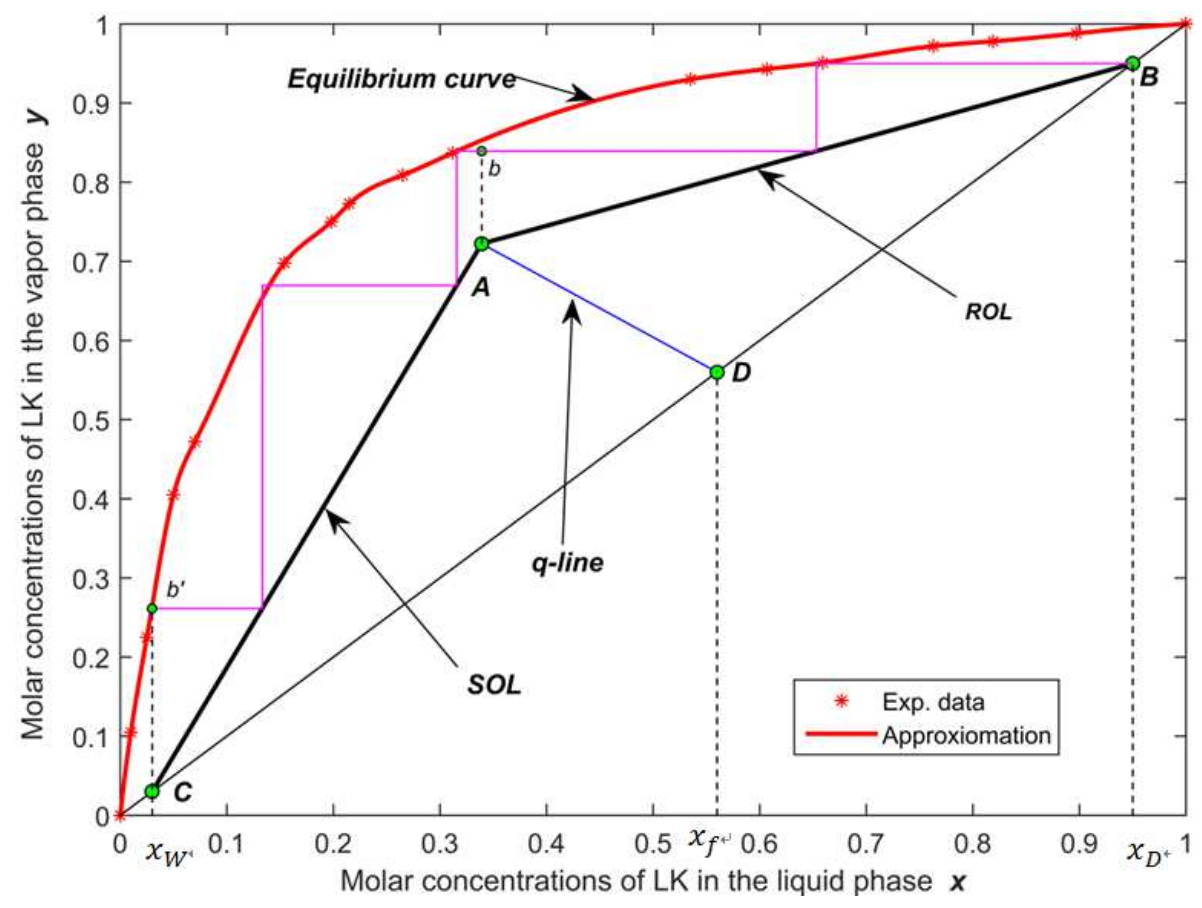

Figure 1. Theoretical stages for the two sections of the column.

\subsubsection{Determination of the Feed Condition (q)}

The locus of the intersections of the operating lines for the enriching section and the stripping section is called the "feed point" and it is obtained by simultaneous solution of the ROL (1) and the SOL (2) equations [17].

$$
y=\left(\frac{q}{1-q}\right) x-\frac{x_{f}}{1-q}
$$

Equation (4) is the equation of a straight line which iscalled the $q$-line. The intersection of the operating lines and $q$-line (point $A$ on Figure 1) corresponds to the feed point. The parameter $q$ is defined by:

$$
q=\frac{H_{V}-H_{\bar{L}}}{H_{V}-H_{L}}=\frac{H_{\bar{F}}}{H_{F}}=\frac{\text { molar heat of vaporization }}{\text { molar latent heat of vaporization }}
$$


where $H_{V}$ is the enthalpy of the vapour, $H_{\bar{L}}$ is the enthalpy of the liquid at its boiling point under standard atmospheric pressure and $H_{L}$ is the enthalpy of the liquid at its entering condition. In equation (5), the parameter $H_{\bar{F}}$ corresponds to the heat required to convert 1 mole of feed from its entering condition to a saturated vapor and $H_{F}$ to the molar latent heat of vaporization of the feed. The values of both $H_{\bar{F}}$ and $H_{F}$ are available; they are calculated in an additive manner shown in section 2.3.1. The parameter $q$ corresponds to the fraction of the feed that is liquid.

For a given feed condition, $x_{f}$ and $q$ are fixed; the slope of the $q$-line is $-\mathrm{q} /(1-\mathrm{q})$ and its intercept $x_{f} /(1-q)$. If $x=x_{f}$, equation (4) results in $y=x_{f}$ and the $q$-line passes through the point $D\left(x_{f}, x_{f}\right)$ on the diagonal. Different values of $q$ will result in a different slope of the $q$ - line.

The construction of the $q$-line is operated as follows; from point $D\left(x_{f}, x_{f}\right)$, a straight line with slope $-q /(1-q)$ is drawn to the intersection with ROL, as shown in Figure 1. The intersection of both lines represents the feed point A. In MatLAB, this is realized using the polyxpoly function, which has the following syntax:

$$
[n, m]=\operatorname{polyxpoly}\left(x_{1}, y_{1}, x_{2}, y_{2}\right)
$$

This function returns the intersection points of two lines in a planar, Cartesian system. In the above equation $\left(x_{1}, y_{1}\right)$ and $\left(x_{2}, y_{2}\right)$ are vectors containing the $\mathrm{x}$ - and $\mathrm{y}$-coordinates of the vertices in the first and second polylines. The output variables, $n$ and $m$, are the $x$ and $y$ coordinates of the intersection of the two lines. In the present case, the intersection between $R O L$ and $q$-line is the feed point $A$. Once the feed point has been determined, the $S O L$ is constructed as a straight line through the points: $C\left(x_{W}, x_{W}\right)$ and $A$. In MatLAB, this is implemented using the polyfit function, which has the following syntax:

$$
z=\operatorname{polyfit}(x, y, 1)
$$

This function returns the coefficients for a polynomial $z(x)$ of degree 1 that is a best fit (in a least-squares sense) for the data in $y$. The coefficients in $z$ are in descending powers,

$$
\mathrm{y}_{2}=0.3728 x_{2}+0.5958=0.3728 \times 0.6532+0.5958=0.8393
$$

The whole first step ends with the determination of point $B_{2}(0.6532,0.8393)$, as shown in Figure 2.

All the necessary steps for the rectifying section of the column are calculated and constructed in the same way. The criterion for completing these calculations is that the coordinate of the last point $x_{n}$ is less than $x_{f}$, because $x_{f}$ is the last point of the rectifying section. Therefore, a loop "while" has to be used in MatLAB to calculate all the steps required for the top of the column.

The theoretical steps for the stripping section of the column are calculated and drawn in a similar way, starting in this case from the last step of the rectifying section and and the length of $z$ is 2 .

After the coefficients of the polynomial have been calculated, the equation (2) or SOL is determined. In the case of the acetone/n-butanol system, equation (2) provides:

$$
y=2.3291 x-0.0372
$$

For the acetone/n-butanol system, the feed temperature of $T_{f}=87^{\circ} \mathrm{C}$ is selected; the results obtained for the equilibrium curve, the operating lines, and the $q$-line are shown in Figure 1.

\subsubsection{Determination of the Number of Theoretical Stages by the McCabe-Tile Method}

According to the McCabe-Tile method, the number of theoretical stages is determined by constructing steps between the working and equilibrium lines [6]. The steps are obtained by sequentially drawing parallel lines to the abscissa and ordinate axes between the working and the equilibrium lines. In order to determine these lines, it is necessary to calculate the coordinates of their end points. For a calculation of the corresponding steps from the top to the bottom of the column, the starting point corresponds to the concentration in the condenser (point $B$ in Figure 1). A line parallel to the abscissa axis is then drawn until it intersects with the equilibrium curve; the upper part of the first step thus starts from point $B\left(x_{D}, y_{D}=x_{D}\right)$ and ends at point $B_{1}$ on the equilibrium curve (see Figure 2). The coordinates of point $B_{1}$ are $\left(x_{1}, y_{1}\right)$, and for the acetone/n-butanol system specifically: $y_{1}=y_{D}=x_{D}=0.95$.

The abscissa of point $B_{1}\left(x_{1}\right)$ represents the equilibrium concentration in the liquid phase of $L K$ corresponding to the vapor concentration $\mathrm{y}_{1}$. This concentration is determined in MatLAB by the combination of spline and ppval functions; these functions (see part 2.1) approximate the liquid-vapor equilibrium. For the acetone/n-butanol system, $x_{1}=0.6532$ and $y_{1}=0.95$ correspond to the coordinates of point $B_{1}$, as illustrated in Figure 2.

The next part of the first stage consists in drawing a line parallel to the ordinate axis from the point $B_{1}$ to the intersection with the ROL at point $B_{2}\left(x_{2}, y_{2}\right)$, with $x_{2}=x_{1}=$ 0.6532 and its ordinate being calculated by equation (3):

incrementing steps until $x_{n}$ becomes less than $x_{W}$. Unlike the previous case, the steps are calculated between the equilibrium curve and equation (6) corresponding to SOL. For the acetone/n-butanol system, the various theoretical stages for both parts of the column are shown in Figure 1.

\subsubsection{Calculation of the Fractional Part of the Last Stage}

Figure 1 displays entire steps for the top section of the column (rectifying section) except for the last one; its end must be calculated as part of the bottom section. The same remark applies for the bottom of the column (stripping section).

Figure $3 \mathrm{a}$ shows the intersection point $A$ of the operating lines and the last theoretical step for the top of the column, 
with the projection of point $A$ on that step (point $\mathrm{b}$ of Figure $3 \mathrm{a}$ and Figure 1). Figure $3 \mathrm{~b}$ similarly shows the projection of point $\mathrm{C}$, which is the end point of the $S O L$ at the bottom of the column, on the bottom last step (point $b^{\prime}$ of Figure $3 b$ and Figure 1).

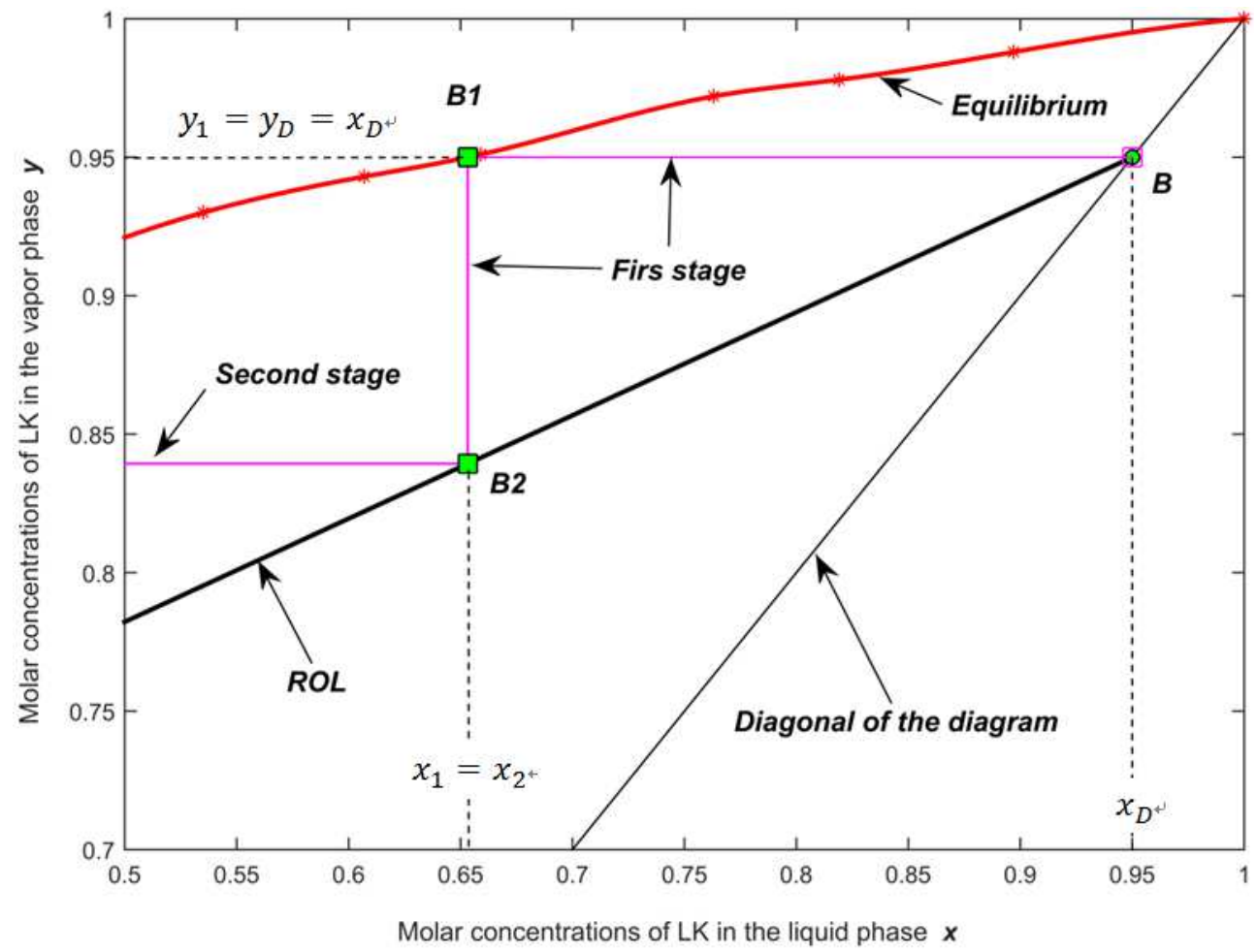

Figure 2. The first theoretical stage.

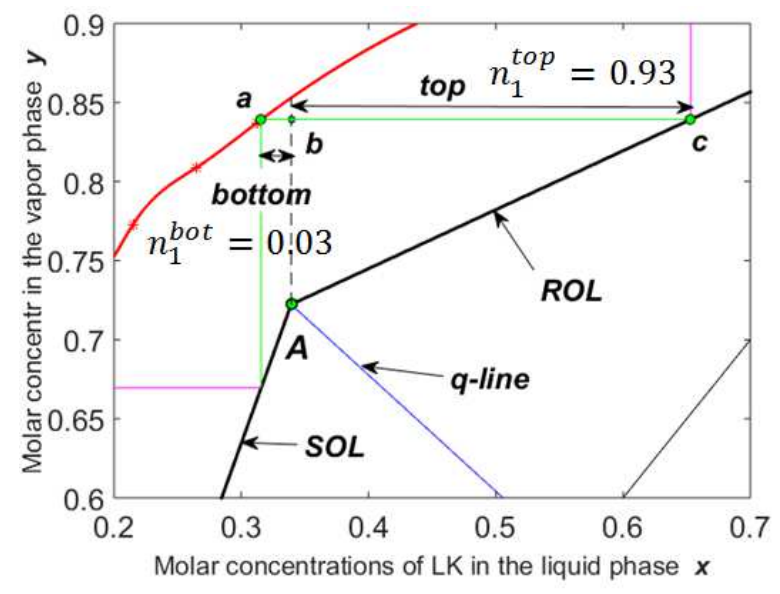

a) Intersection of the operating lines

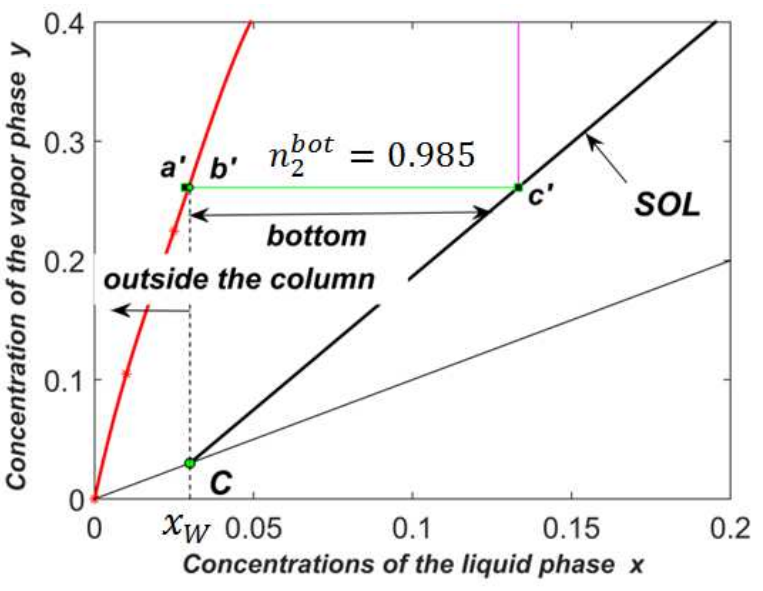

b) Endpoint of the stripping operating line

Figure 3. Determination of the last steps for both parts of the column.

From point $A$, a straight line, parallel to the ordinate, rises to the intersection with the last step of the rectifying section, resulting in a dashed section $\overline{A b}$ (see Figure $3 \mathrm{a}$ ).

The entire step represents the segment $\overline{a c}$; the part of this step corresponding to the top of the column is the segment $\overline{b c}$ while he segment $\overline{a b}$ represents the part corresponding to the bottom of the column. The lengths of the segments $\overline{a c}$ and $\overline{b c}$ are measured. For example, if $\overline{a b}=12 \mathrm{~cm}$ and $\overline{b c}=7 \mathrm{~cm}$, the part of the $8^{\text {th }}$ step corresponding to the rectifying section of the column is:

$$
n_{1}^{t o p}=\frac{\overline{b c}}{\overline{a b}}=\frac{7}{12}=0.5833 \approx 0.6
$$

In MatLAB, this is represented by the difference in the abscissa of the last three points $-a\left(x_{1}\right), b\left(x_{A}\right)$ and $c\left(x_{2}\right)$, the abscissa of point $b\left(x_{A}\right)$ being calculated by the $q$-line equation.

For the acetone/n-butanol system, the part of the second step that corresponds to the rectifying section is $n_{1}^{\text {top }}=0.93$. For this section, there is one entire step: $N^{t o p}=1$ (see 
Figure 1). The total number of theoretical stages for the rectifying section is therefore:

$$
N_{t}^{\text {top }}=N^{\text {top }}+n_{1}^{\text {top }}=1+0.93=1.93
$$

The portion of the step corresponding to the segment $\overline{a b}$ can be determined as follows:

$$
n_{1}^{b o t}=1-n_{1}^{t o p}=1-0.93=0.07
$$

This part is added to the number of theoretical stages for the stripping section of the column.

It is similarly proceeded at the end of the SOL, as shown in Figure $3 b$. The entire last step corresponds to the segment $\overline{a^{\prime} c^{\prime}}$ while the segment $\overline{b^{\prime} c^{\prime}}$ corresponds to the part of this step for the stripping section that can be determined as described above. For the acetone/n-butanol system, the following is obtained:

$$
n_{2}^{\text {bot }}=0.985
$$

There is one entire step for the stripping section, i.e. $N^{b o t}=1$, and the total number of stages is:

$$
N_{t}^{b o t}=N^{b o t}+n_{1}^{b o t}+n_{2}^{b o t}=1+0.07+0.985=2.055
$$

\subsection{Ponchon-Savarit Method}

The assumptions of constant molar overflow in distillation seriously curtail the general utility of the McCabe-Thiele method. This disadvantage is avoided in the Ponchon-Savarit method. The operating lines are developed from enthalpy balances on the rectifying and stripping sections in the $\mathrm{H}-\mathrm{y}-\mathrm{x}$ diagram [7-9]. This graphical method uses both material and energy balances to determine the number of theoretical stages.

\subsubsection{Calculation and Approximation of the Saturated Enthalpy Curves in Both Phases}

In order to construct the $H-y-x$ diagram, three parameters are required: $H$ - enthalpies; $y$ - the molar concentration in the vapor; $x$ - molar concentration in the liquid.

The parameters $x$ and $y$ are available and they are determined by the data equilibrium liquid-vapor (see Part 1). The enthalpies in both phases are unknown: $H_{v}$ - saturated vapor enthalpies and $H_{l}$ - saturated liquid enthalpies. To determine these enthalpies, the latent-heat enthalpy model is used [18]. Thus, for each experimental liquid-vapor equilibrium point, the enthalpies $H_{v}$ and $H_{l}$ are calculated; they constitute "experimental enthalpy data" because they are obtained directly from the vapor-liquid equilibrium data. To determine $H_{v}$ and $H_{l}$ at any point of the composition range, the enthalpy functions shall be determined. The enthalpy functions have been generated from the fittings of the experimental enthalpy data in all the composition range to the appropriate functions. In order to produce enthalpy functions, the polynomial form of the cubic spline interpolant obtained by "sline" functions in MatLAB has been used.

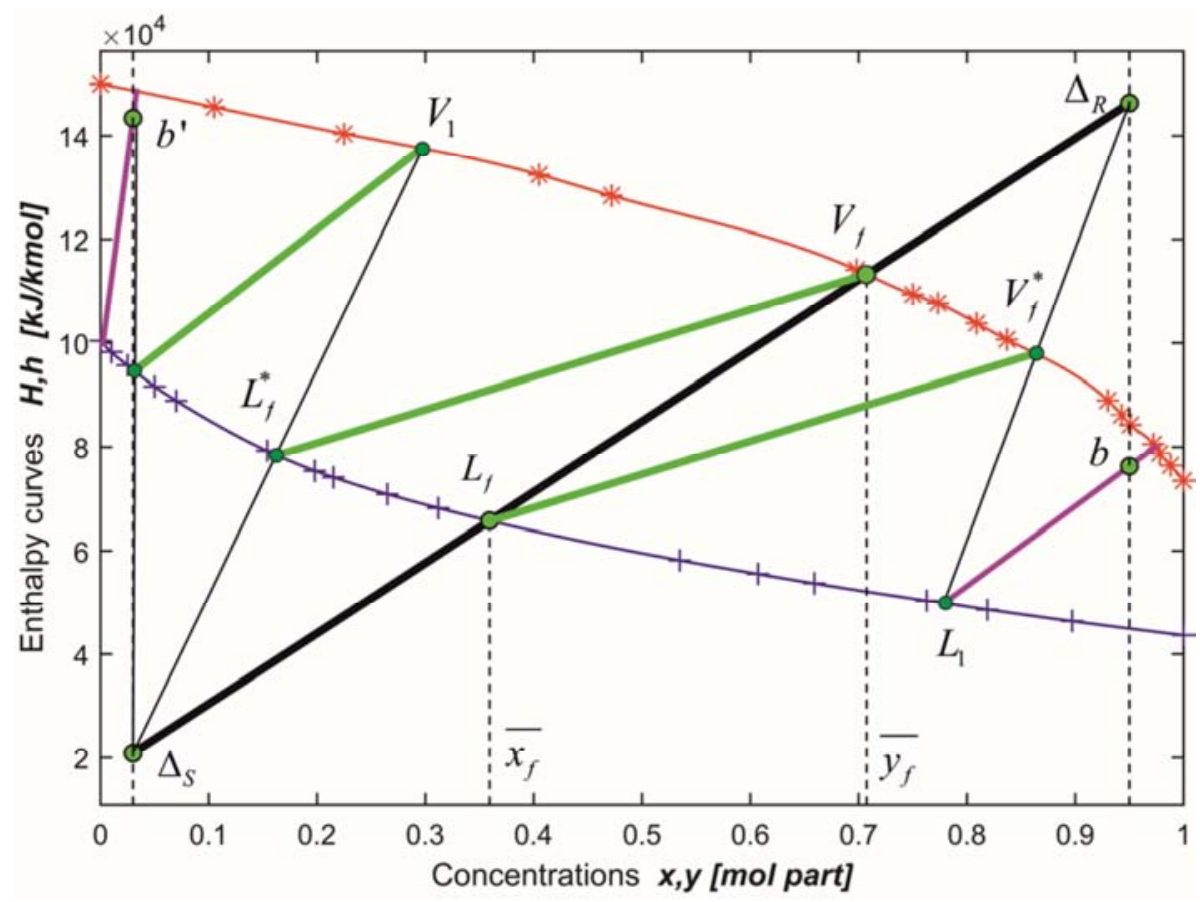

Figure 4. Graphical presentation of the number of stages for the rectifying section.

Figure 4 shows experimental enthalpy data corresponding to the liquid "+" and vapor "*" phase respectively as well as the saturated vapor curve (red curve) and the saturated liquid curve (blue curve) obtained by enthalpy functions for the acetone/n-butanol system. The figure shows a good agreement between experimental and calculated results.

\subsubsection{Determination of the Difference Points Line}

The next step in the Ponchon-Savarit method consists in locating the "difference points line", which is a straight 
line between two operating points called "difference points". The rectifying section difference point $\Delta_{R}$ has its $x$ coordinate equal to the concentration of $L K$ in the condenser $-\Delta_{R}\left(x_{D}, h_{D}^{\prime}\right)$, and the stripping section difference point $\Delta_{S}$ has its $x$ coordinate equal to the concentration of $L K$ in the reboiler $-\Delta_{S}\left(x_{W}, h_{W}^{\prime}\right)$. According to the Ponchon-Savarit method [7-9], the difference points line coincides with the 'feed line', which is equivalent to the $q$-line in the McCabe-Tile method. This line is defined by two points labeled $L_{f}$ and $V_{f}$; he point $L_{f}\left(\overline{x_{f}}, H_{l f}\right)$ represents the enthalpy of the liquid phase corresponding to the concentration of $L K$ in the feed stage $\overline{x_{f}}$ and the point $V_{f}\left(\overline{y_{f}}, H_{v f}\right)$ the enthalpy in the vapor phase corresponding to the working vapor concentration $\overline{y_{f}}$ in the feed stage. Using the $q$-line equation (5), both $\overline{x_{f}}$ and $\overline{y_{f}}$ can be determined (see part 2.2.2) and allows for the enthalpies $H_{l f}$ and $H_{v f}$ to be calculated. The point with coordinates $\left(\overline{x_{f}}, \overline{y_{f}}\right)$ actually corresponds to point $A$ in Figure 1. The enthalpy curves $H_{v}=f(y)$ and $H_{l}=f(x)$ are used andthe feed line $\overline{L_{f}, V_{f}}$ is defined and shown for the acetone/n-butanol system in Figure 4.

According to the Ponchon-Savarit method, points $\Delta_{R}, L_{f}, V_{f}$ and $\Delta_{S}$ are colinear, i.e. the feed line and the difference points line match; the segment $\overline{L_{f} V_{f}}$ can thus be extended until it intersects with the perpendiculars elevated from the points $x_{D}$ and $x_{W}$ at points $\Delta_{S}$ and $\Delta_{R}$ respectively. In the MatLAB, this is done as follows:

1. For the two points $L_{f}$ and $V_{f}$ the polyfit function determines the coefficients for a polynomial of $z 1(x)$ of degree 1 passing through the two points.

2. The polyfit function determines the coefficients for a polynomial of $z 2(x)$ of degree 1 that is parallel to the ordinate and passes through a point with coordinates $\left(x_{D}, 0\right)$.

3. The polyfit function determines the coefficients for a polynomial of $z 3(x)$ of degree 1 that is parallel to the ordinate and passes through a point with coordinates $\left(x_{W}, 0\right)$.

4. The polyxpoly function finds the coordinates of the intersection points of two lines $z 1(x)$ and $z 2(x)$. This is the coordinate of the rectifying section difference point $\Delta_{R}$.

5. The polyxpoly function finds the coordinates of the intersection points of two lines $z 1(x)$ and $z 3(x)$. This is the coordinate of the rectifying section difference point $\Delta_{W}$

For the acetone/n-butanol system, the points $\Delta_{R}, L_{f}, V_{f}, \Delta_{S}$ and the difference point line are shown in Figure 4.

\subsubsection{Determining the Number of Stages}

The number of theoretical plates is then determined by application of the straight line relationship on the $H-x-y$ diagram and the equilibrium data.

\section{Rectifying section}

Using the sline function for an approximation of the liquid-vapor equilibrium (see part 1), the equilibrium concentration $\overline{y_{f}^{*}}$ corresponding to a liquid concentration of $L K$ in the feed stage $\overline{x_{f}}$ is determined.

By means of the enthalpy curves $H_{v}=f(y)$, the enthalpy $H_{y 1}$ corresponding to the vapor concentration $\overline{y_{f}^{*}}$ is calculated, as illustrated by the point $V_{f}^{*}\left(\overline{y_{f}^{*}}, H_{y 1}\right)$ in Figure 4. The points $L_{f}$ and $V_{f}^{*}$ represent the two ends of the line segment $\overline{L_{f} V_{f}^{*}}$ (green line in Figure 4).

The segment $\overline{\Delta_{R} V_{f}^{*}}$ connects the rectifying section difference point $\Delta_{R}$ with the point $V_{f}^{*}$. According to the Ponchon-Savarit method, the extension of this segment to its intersection with the liquid enthalpy curve $H_{l}=f(x)$ (point $L_{1}$ on the Figure 4) corresponds to the first stage in the rectifying section of the column. Thereby, the segment $\overline{\Delta_{R} V_{f}^{*} L_{1}}$ is a straight line, which connects the rectifying section difference point $\Delta_{R}$ to point $L_{1}$. In MatLAB, this is realized using the polyfit and polyxpolyfunctions:

1. Polynomial $g(x)$ of degree 1 passing through the points $\Delta_{R}$ ang $V_{f}^{*}$ using the polyfit function is defined.

2. The polyxpoly function finds the coordinates of the intersection points of two polinomials $g(x)$ and liquid enthalpy curve $H_{l}=f(x)$. This is the coordinate of the point $L_{1}\left(x_{1}, H_{x 1}\right)$.

3. The green polyline $\overline{L_{f} V_{f}^{*} L_{1}}$ in Figure 4 correspond to the first stage of the rectifying section.

Similarly, starting from point $L_{1}$, all other steps for the rectifying section are determined. As with the McCabeThiele method (see part 2.2.4), the last step in the PonchonSavarit method for the rectifying section is not entire (see point $b$ in Figure 4). The fractional part of this last stage is calculated in the same way as in the McCabe-Thiele method (see part 2.2.4). For the acetone/n-butanol system, the number of theoretical stages for the rectifying section determined by the Ponchon-Savarit method is $N_{t}^{t o p}=$ 1.87722 .

\section{Stripping section}

The number of theoretical stages for the stripping section of the column can be similarly found. The difference relates to the starting point which corresponds to the concentration of $L K$ in the vapor phase of the feed stage $\overline{y_{f}}$, as illustrated by point $V_{f}$ in Figure 4.

With the sline function for the liquid-vapor equilibrium approximation (see part 1 ), the equilibrium concentration $\overline{x_{f}^{*}}$ corresponding to the vapor concentration of $L K$ in the feed stage $\overline{y_{f}}$ can be determined. By using the enthalpy curves $H_{l}=f(x)$, the enthalpy $H_{x 1}$ corresponding to the concentration $\overline{x_{f}^{*}}$ is calculated, as illustrated by point $L_{f}^{*}\left(\overline{x_{f}^{*}}, H_{x 1}\right)$. The points $V_{f}$ and $L_{f}^{*}$ represent the two ends of the line segment $\overline{V_{f} L_{f}^{*}}$ (green line in Figure 4). The segment $\overline{\Delta_{S} L_{f}^{*}}$ connects the stripping section difference point $\Delta_{S}$ with the point $L_{f}^{*}$. According to the Ponchon-Savarit method, the extension of this segment to its intersection with the vapor enthalpy curve $H_{v}=f(y)$ (point $V_{1}$ on the Figure 4) corresponds to the first stage in the stripping section of the column. The segment $\overline{\Delta_{s} L_{f}^{*} V_{1}}$ is the straight line connecting the stripping section difference point $\Delta_{S}$ to the point $V_{1}$. In 
MatLAB, this is realized in the same way as in the rectifying section by the combination between polyfit and polyxpoly functions. Therefore, the green polyline $\overline{V_{f} L_{f}^{*} V_{1}}$ correspond to the first stage of the stripping section. Similarly, starting from the point $V_{1}$, all other steps for the stripping section are determined. The fractional part of this last stage (see point $b^{\prime}$ in the Figure 4 ) is calculated in the same way as in the rectifying section. For the acetone/nbutanol system the number of theoretical stages for stripping section determined by the Ponchon-Savarit method is $N_{t}^{\text {top }}=2.2299$.

\subsection{Determination of Optimum Reflux Ratio}

Economic calculations are important in industrial designs. The total cost of a distillation unit includes capital costs for the equipment as well as installation and operating costs for utilities, labor, raw materials, and maintenance and energy requirements. The desired separation can be achieved with relatively low energy requirements by using a large number of trays, thus incurring larger capital costs with the reflux ratio at its minimum value. On the other hand, by increasing the reflux ratio, the overhead composition specification can be met by a fewer number of trays but with higher energy costs. Thus, the minimum of the total cost should correspond to the optimal reflux ratio $R_{\text {opt }}$ [19].

The steam required will be proportional to the molar vapor flow in the column $V_{v}$. Under the consideration of McCabeThiele method first the concept of total condenser is used and second in the column for every mole of liquid vaporized, a mole of vapor is condensed, thus $V_{v}$ is constant along the column. In a total condenser, the entire vapor leaving the top of the column $V_{v}$ is condensed. One part of $V_{v}$ is returned to the column as reflux $V_{F}$ and another is separated as a liquid distillate product $V_{D}$ i.e. $V_{v}=V_{F}+V_{D}$. The reflux ratio is $R=V_{F} / V_{D}$ then $V_{v}=V_{D}(R+1)$. Therefore, the steam required per mole of product is proportional to $(R+1)$, minimal when $R$ equals $R_{\min }$ and steadily rising as $R$ increases. The molar vapor flow in the column $V$ being proportional to the column diameter $D, D$ is proportional to $(R+1)$.

A reduction in the required number of stages $N$ as $R$ is increased beyond $R_{\min }$ will tend to reduce the cost of the column. At values still close to $R_{\text {min }}$, a marked reduction in the number of stages can be attained, although at higher values of $R$, further increases have little effect on the number of plates [20].

The total volume of the column is $V=D H$, where $H$ is the height of the column. The height of the column is proportional to $N$ and then $V$ is proportional to $N(R+1)$. The objective function $F=N(R+1)$ is thus defined, with the minimum of this function corresponding to the minimum in the volume of the column. This volume criterion can be used to determine the optimum reflux ratio $R_{o p t}$.

The MatLAB code presented above for both the McCabe-Thiele and Ponchon-Savarit methods is running with a fixed reflux ratio. Thus, if a series of reflex ratios $R_{i}$ are selected, for each of them, the number of theoretical degrees $N_{i}$ can be determined by the two graphical methods. Then, the minimum of the function $R=f(F)$ is found. Thereby, the reflux ratio corresponding to $\min (F)$ represents the optimum reflux ratio $R_{\text {opt }}$, which allows minimizing the volume of the column and thereby reducing the total cost of a distillation unit. A smaller column volume means:

1. Lower column height, respectively fewer theoretical stages - minimizes the capital costs;

2. Smaller column diameter, respectively smaller molar vapor flow in the column $V_{v}$ - minimizes operating costs.

Rules of thumb are often used by designers to shortcut reflux optimization. Such rules of thumb are usually expressed as the optimum reflux ratio of minimum reflux $R_{\text {opt }} / R_{\text {min }}$.

According to a number of authors [20-23], $R_{\text {opt }} / R_{\min }$ ranges from $1.1-1.5$ times to the minimum. It should be emphasized, however, that in many cases, much higher values of $R_{\text {opt }} / R_{\min }$ are being employed, especially in the case of vacuum distillation. In this study, the range of variation of reflux ratio from $1,1 R_{\min }$ to $10 R_{\min }$ has been adopted.

According to the theory of both graphical methods [6-9], the minimum reflux ratio $R_{\min }$ can be calculated as follows:

$$
R_{\min }=\frac{x_{D}-y_{e q}^{*}}{x_{D}-x_{e q}}
$$

The concentration $y_{e q}^{*}$ representing the equilibrium concentration corresponds to the $x_{e q}$ concentration of point A. It is calculated using the $q$-line function (see part 2.2.2) and the already defined spline function characterizing the vapor-liquid equilibrium. For the acetone/n-butanol system, it is obtained: $R_{\text {min }}=$ 0.285235 . Thus, varying the reflux ratio with the volume criterion defined above and using McCabe-Thiele or Ponchon-Savarit methods, the optimum reflux ratio is determined. The result of applying the above procedure to the acetone/n-butanol system is presented in Figures 5a and $5 b$.

Figure 5 shows a clear minimum of the objective function in both cases. The optimum reflux ratios obtained by McCabe-Thiele and Ponchon-Savarit methods are $R_{\text {opt }}=$ 0.594407 and $R_{\text {opt }}=0.696462$ respectively.

To determine the theoretical number of stages and the optimum reflux ratio according to McCabe-Thiele or Ponchon-Savarit methods, the following algorithm is proposed:

1. Initial data: experimental liquid/vapor equilibrium data, data for enthalpy calculation, $x_{D}, x_{f}, x_{W}, T_{f}$;

2. Equilibrium data approximation;

3. $q$ - line determination;

4. $R_{\min }$ determination;

5. Formation of $R$ vector from $1,1 R_{\text {min }}$ to $10 R_{\text {min }}$;

6. Selecting a method: McCabe-Thiele or PonchonSavarit;

7. Determination of number of stages for all elements of 
$R$ vector by selected method;

8. Defining the objective function: $F=N(R+1)$;

9. Determining the minimum of the objective function $F$;

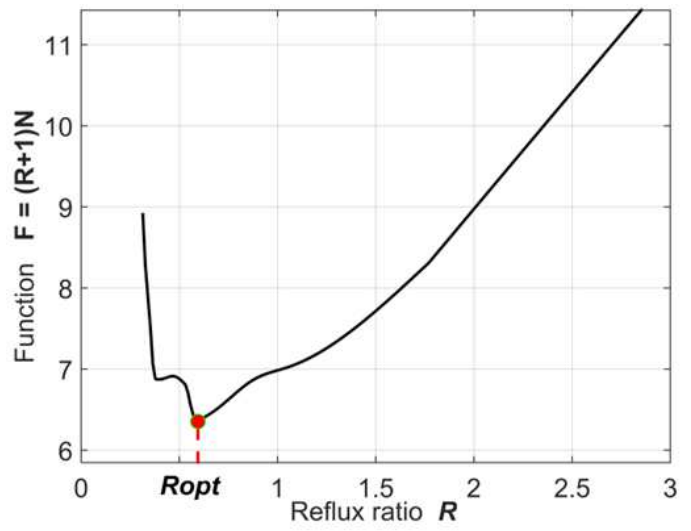

a) McCabe-Thiele method
10. Determining the $R_{o p t}$;

11. Determining the number of stages using $R_{\text {opt }}$;

12. Plot and printing of final results.

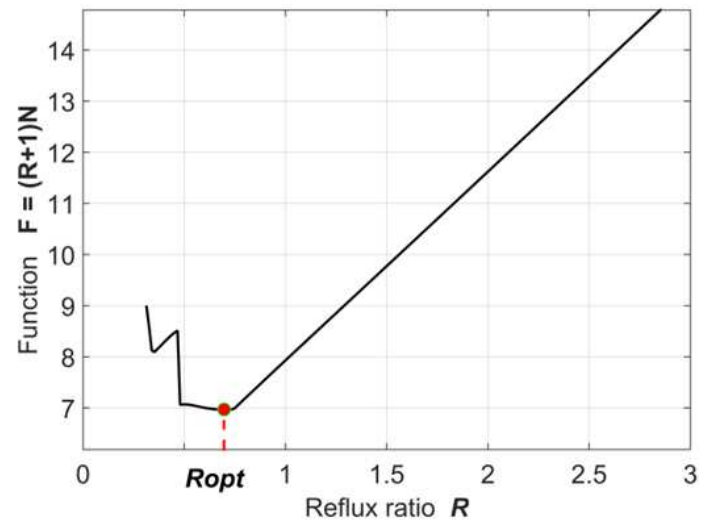

b) Ponchon-Savarit method

Figure 5. Graphical results of the optimal reflux ratio for the acetone/n-butanol system.

\section{Results and Discussion}

Using the acetone/n-butanol system, the individual steps in MatLAB code have been developed. Figures 1 and 4 graphically show the theoretical number of stages obtained for this system by the McCabe-Thiele and Ponchon-Savarit methods, respectively. Figures $5 \mathrm{a}$ and $5 \mathrm{~b}$ graphically present the results for determining the optimum reflux ratio by both methods. The final results are summarized in Table 1 .

The sensitive analyses for number of working reflux ratios $R_{i}$ in the range of $1,1 R_{\text {min }}$ to $10 R_{\text {min }} 10$ have been made.
The number of $R_{i}$ was varied from 10 to 1000; it was found that for $R_{i}>200$, no changes in the determination of $R_{o p t}$ occurred. Thus, in this study, $R_{i}=200$ was used. Despite this result, the MatLAB code provides an opportunity to set the number of working reflux ratios $R_{i}$. Table 1 shows that for the acetone/n-butanol system, the two graphical methods give almost identical results. With regard to the total number of stages, $N \approx 4$ is obtained by both methods. There is little difference with respect to the optimal reflex number: McCabe-Thiele $R_{\text {opt }}=0.6$, Ponchon-Savarit $R_{\text {opt }}=0.7$.

Table 1. Results for acetone/n-butanol system.

\begin{tabular}{lll}
\hline Parameter & McCabe-Thiele & Ponchon-Savarit \\
\hline Minimum reflux ratio & 0.2852 & 0.2852 \\
Optimum reflux ratio & 0.594407 & 0.696462 \\
Number of theoretical stages for rectifying section & 1.93033 & 1.87722 \\
Number of theoretical stages for stripping section & 2.05526 & 2.2299 \\
Total number of theoretical stages & 3.98559 & 4.10713 \\
Top $L K$ mass fraction & 0.95 & 0.95 \\
Bottom $L K$ mass fraction & 0.03 & 0.03 \\
\hline
\end{tabular}

Table 2. Results for the acetone/n-butanol system.

\begin{tabular}{lll}
\hline Parameter & FUG with Fenske feed stage location & FUG with Kirkbride feed stage location \\
\hline Minimum reflux ratio & $2.77 \times 10^{-1}$ & $2.77 \times 10^{-1}$ \\
Optimum reflux ratio & $7.77 \times 10^{-1}$ & $7.77 \times 10^{-1}$ \\
Total number of theoretical stages & 6.43 & 6.43 \\
Feed stage location & 3.92 & 4.24 \\
Top $L K$ mass fraction & $9.76 \times 10^{-1}$ & $9.76 \times 10^{-1}$ \\
Bottom $L K$ mass fraction & $6.16 \times 10^{-2}$ & $6.16 \times 10^{-2}$ \\
\hline
\end{tabular}

In order to verify the possibility of using both graphical methods for SHORT-CUT distillation design, the acetone/nbutanol system has been modeled by conventional SHORTCUT methods. To perform this simulation, the ChemCAD 7.1.5 simulator has been used. The shortcut distillation module in the simulator uses the Fenske-Underwood-Gilliland (FUG) method $[4,24,25]$ to simulate a simple distillation column. The feed location can be calculated by the Fenske [24] or
Kirkbride [26] equations. In order to determine the thermodynamic of the system, the same experimental data for the vapor/liquid equilibrium [15] and NRTL model have been used [27, 28]. The use of ChemCAD allowed regress experimental data and generate binary interaction parameters (BIP) for mixtures. The results of the simulation for the acetone/n-butanol system are shown in Table 2.

The only difference between the two methods in the 
ChemCAD shortcut distillation module is about the feed stage location. The results are however very close for this parameter and basically show the same feed stage location $N_{f} \approx 4$.

In the FUG method, the reflux ratio $R$ or the ratio $R / R_{\min }$ must be specified. Thus, there is no possibility to obtain the optimum reflux ratio. If $R / R_{\text {min }}$ is set, ChemCAD first calculates $R_{\text {min }}$ and then determines $R_{\text {opt }}$ as $R / R_{\text {min }}$ ratio. Determination of $R_{\min }$ in the ChemCAD shortcut distillation module is performed by the Underwood procedure [25].

The resulting value is $R_{\min }=0.277358$ whereas $R_{\text {min }}=$ 0.285235 is obtained if using graphical methods. The two values are very close, which is a criterion showing the ability to use graphical methods for SHORT-CUT distillation design.

In the ChemCAD shortcut distillation module, a case study option is provided to allow to vary $R / R_{\text {min }}$ in a specified range and review its effect on column performance indicators such as the number of stages or the final product concentration.

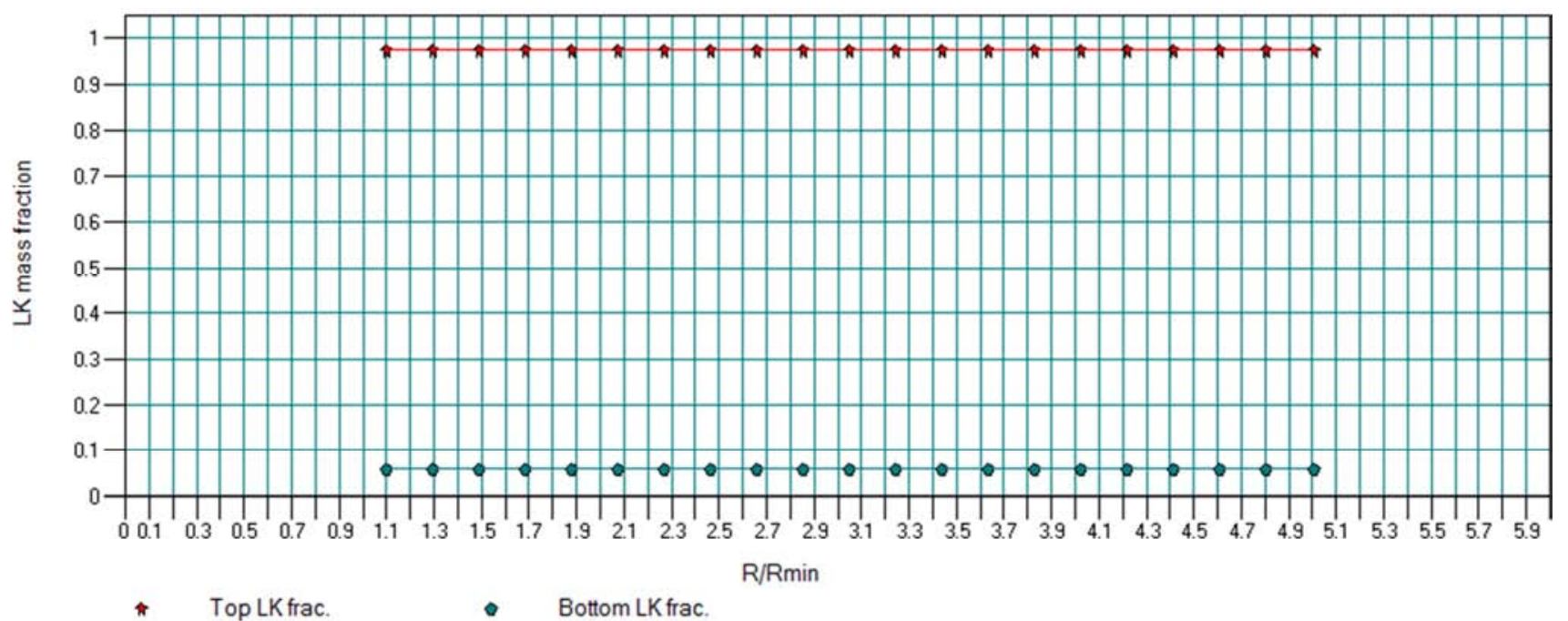

Figure 6. Sensitive study for influence of $R / R_{\min }$ on the final $L K$ concentration.

On Figure 6, the sensitive analyses to check the influence of $R / R_{\min }$ on the final $L K$ mass fraction in the distillate and in the bottom product are shown. The figure shows that the change in $R / R_{\text {min }}$ does not affect the final $L K$ mass fraction. The desired value of $L K$ mass fraction in the distillate is $x_{D}=0.95$ whereas the value of this parameter obtained by ChemCAD shortcut module is $x_{D}=0.98$. The desired value of $L K$ mass fraction in the bottom product is $x_{W}=0.030$ whereas the value of that parameter obtained by ChemCAD shortcut module is $x_{W}=0.062$. Thus, according to the FUG SHORT-CUT method, the separation of the acetone $/ \mathrm{n}$ butanol system by rectification makes it impossible to obtain the bottom product with $L K$ mass fraction $x_{W}=0.03$.

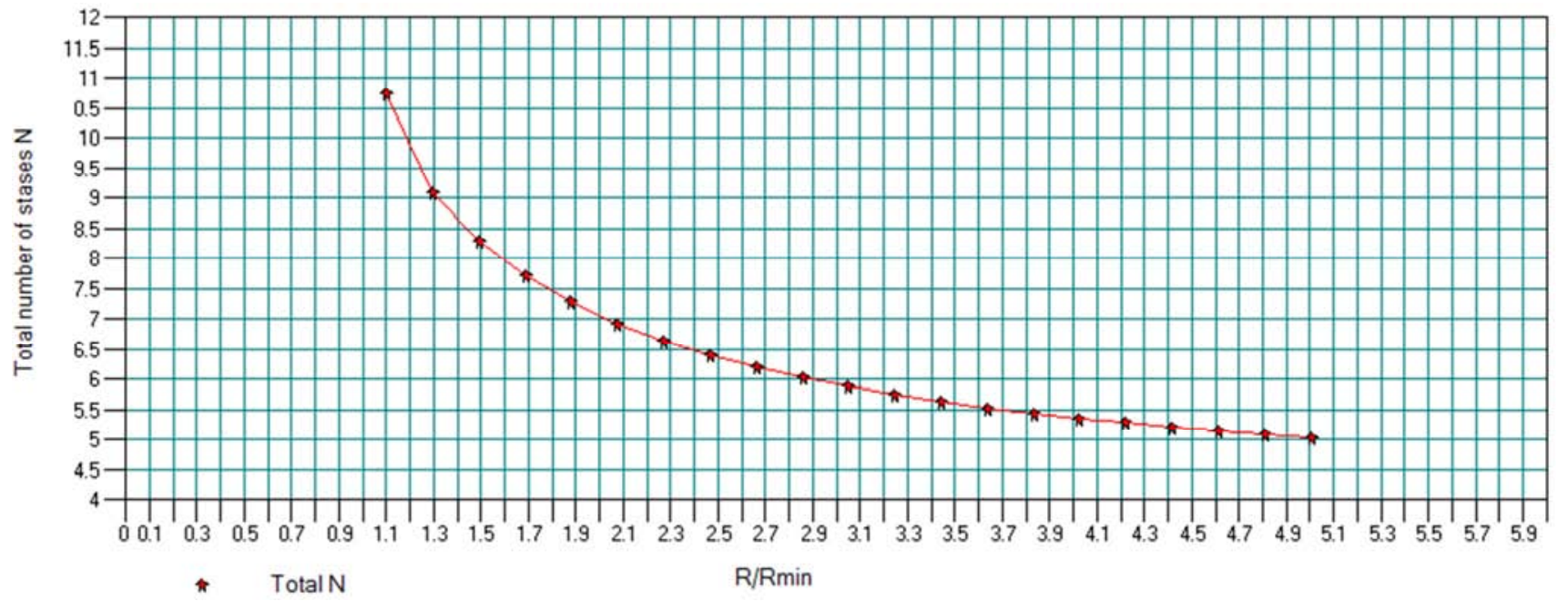

Figure 7. Sensitive study for influence of $R / R_{\min }$ on the total number of stages.

On Figure 7 the sensitive analyses to check the influence of $R / R_{\min }$ on the total number of stages is shown. It can be seen from the figure that after $R / R_{\min }>2.8$, the reduction of the total number of stages is very slight. Thus, $R / R_{\min }=$ 2.8 is the optimum ratio and the value $R_{\text {opt }}=0.78$ for the optimum reflux ratio is then obtained. Determining $R_{\text {opt }}$ in 
this way is however not very accurate because it takes into account the influence of $R$ only on the total number of stages but not on the total cost of a distillation unit. Therefore, defining $R_{\text {opt }}$ using the MatLAB code, as proposed here, has an advantage over the FUG method for the following two main reasons:

1. The sensitive-analysis option is additional and not integrated into the FUG SHORT-CUT method itself. Thus, after determining the number of stages, further research must be conducted to determine $R_{\text {opt }}$, whereas the MatLAB code suggested here directly determines $R_{\text {opt }}$ based on the volume criteria (see part 2.4);

2. Determination of $R_{\text {opt }}$ by sensitive analyses in the FUG SHORT-CUT method does not take into account the total cost of a distillation unit whereas the volume criteria in the MatLAB code (see function $F=N(R+1)$ in part 2.4) account for the total cost of a distillation unit. Therefore, defining $R_{\text {opt }}$ is much more accurate.

For comparison to the SHORT-CUT methods proposed here (graphical and FUG methods), a rigorous calculation has been made by using the SCDS module in the ChemCAD simulator.

SCDS is a rigorous multi-stage vapor-liquid equilibrium module which simulates any single column calculation [13]. This module is mainly designed to simulate non-ideal $K$-value chemical systems. It uses a Newton-Raphson convergence method and calculates the derivatives of each equation rigorously.

The calculation algorithm of the SCDS module requires to initially setting the number of theoretical stages and the feed stage location. Then as a result of the solution the concentration of LK in the top and the bottom of the column is obtained. In this case, in order to make a comparison between the rigorous and SHORT-CUT methods, the number of theoretical stages 5 and the feed stage location 3 are selected. SCDS offers a variety of specifications, such as total mole flow rate, heat duty, reflux ratio, boil-up ratio, temperature etc. Therefore, there are several different ways to initially define the SDST module. The results obtained by SCDS modulates for the acetone/n-butanol system are presented in Table 3.

Table 3. Rigorous results obtained for the acetone/n-butanol system via the SCDS module.

\begin{tabular}{ll}
\hline Parameter & SCDS module \\
\hline Reflux ratio & 0.7 \\
Total number of theoretical stages & 5 \\
Feed stage location & 3 \\
Top $L K$ mass fraction & 0.95149058 \\
Bottom $L K$ mass fraction & 0.03 \\
\hline
\end{tabular}

The results in Table 3 show that there is little difference between the SHORT-CUT and the rigorous calculation. For comparison in terms of accuracy between the graphical methods and the FUG method in ChemCAD shortcut distillation module, the differences are indicated: on the one hand between the FUG method and the rigorous calculation, on the other hand between graphical methods and the rigorous calculation.

1. The rigorous calculations show that the desired end results are achieved by the reflux ratio $R=0.7$. This value is closer to the optimum reflux ratio obtained by the Ponchon-Savarit method rather than by the FUG method;

2. The rigorous calculation gives a total number of 5 theoretical stages. For this parameter, the graphical methods give 4 while the FUG method gives 6 . The difference between the rigorous calculation and all SHORT-CUT methods is thus the same;

3. The rigorous calculations show that it is possible to obtain distillate with the $L K$ mass fraction $x_{D}=$ 0.95149058 , and the bottom product with the LK mass fraction $x_{W}=0.03$. According to the results of the graphical methods (see Table 1), this is possible whereas the sensitive analyses of the FUG method (see Figure 6) show that this is not possible. According to Figure 6, the minimum bottom $L K$ mass fraction is $x_{W}=$ 0.06156551 . Thus, with respect to the bottom and top $L K$ mass fraction, the results of graphical methods practically coincide with the rigorous calculation.

\section{Conclusion}

A MatLab code for SHORT-CUT distillation design using the McCabe-Thiele and Ponchon-Savarit methods is proposed, in the first place because those methods are easy to apply and are not time consuming, and moreover because they allow for the easy visualization of the interrelationships among variables. By the combination of the two functions spline and ppval, experimental vapor-liquid equilibrium data and enthalpy data for both phases have been approximated. In the studied system, this approximation has given very accurate results. In order to obtain a basic estimation of both methods, a combination of several MatLAB functions such as "polyxpoly", "polyfit", "min", and "max"has been used.

The volume criterion has been defined and used to determine the optimum reflux ratio $R_{\text {opt }}$, which allows minimizing the volume of the column and thereby reducing the total cost of a distillation unit. The sensitive analyses for number of working reflux ratios $R_{i}$ in the range of $1,1 R_{\min }$ to $10 R_{\text {min }} 10$ have been made. The number of $R_{i}$ was varied from 10 to 1000 . It was found that for $R_{i}>200$, no changes in the determination of $R_{\text {opt }}$ occurred. Thus, in this study, $R_{i}=200$ has been used. Notwithstanding this result, the MatLAB code provides an opportunity to set the number of working reflux ratios $R_{i}$.

To evaluate the accuracy of the results obtained with the graphical methods via the generated MatLab code, a comparison with the results calculated by other SHORT-CUT methods and rigorous calculations has been performed. The ChemCAD 7.1.5 simulator has been used for this comparison; its shortcut distillation module uses the FenskeUnderwood-Gilliland (FUG) method. For rigorous estimation, the SCDS multi-stage vapor-liquid equilibrium 
module has been used.

The results show that the graphical methods are closer to the results of rigorous calculations than the FUG SHORTCUT method. This is both with respect to the reflux ratio and to the bottom and top $L K$ mass fraction. It can therefore be concluded that the McCabe-Thiele and Ponchon-Savarit graphical methods can be successfully used for SHORT-CUT distillation design. The main advantage of these methods over the FUG method in the ChemCAD shortcut distillation module is the criterion defined in Part 2.4 to determine the optimal reflux ratio.

\section{Acknowledgements}

The authors are grateful to « Invited Fellow protocol » of University Sorbonne Paris Nord which has contributed to enhance the collaboration of joint innovative research between UCTM Sofia and LSPM CNRS UPR3407.

\section{References}

[1] Kong L, Maravelias CT (2020). Generalized short-cut distillation column modeling for superstructure-based process synthesis. AIChE J., 66 (2). https://doi.org/10.1002/aic.16809

[2] Adiche Ch., Vogelpohl A. (2011). Short-cut methods for the optimal design of simple and complex distillation columns. ChERD, 89 (8), 1321-1332.

[3] Zubira M. A. et al. (2019). Economic, Feasibility, and Sustainability Analysis of Energy Efficient Distillation Based Separation Processes. Chem. Eng. Transactions, 72, 109-114.

[4] Gilliland E. R. (1940). Multicomponent Rectification Estimation of the Number of Theoretical Plates as a Function of the Reflux Ratio. Ind. Eng. Chem., 32 (9), 1220-1223.

[5] Erbar J. H., Maddox R. N. (1961). Latest score: reflux vs. Trays. Pet. Ref., 40 (5), 183.

[6] McCabe W. L, Thiele W. E (1925). Graphical Design of Fractionating Columns. Ind. Eng. Chem., 17 (6), 605-611.

[7] Ponchon M. (1921). Graphical study of distillation. Tech. Modern, 13, 20.

[8] Savarit R. (1922). Definition of Distillation, Simple Discontinuous Distillation, Theory and Operation of Distillation Column, and Exhausting and Concentrating Columns for Liquid and Gaseous Mixtures and Graphical Methods for Their Determination. Arts et Metiers, 3, 65.

[9] Mohapatro R. N. et al. (2021). Separation Efficiency Optimisation of Toluene-Benzene Fraction using Binary Distillation Column. J. Inst. Eng. India Ser., D 102, 125-129.

[10] Seedat N., Kauchali Sh., Patel B. (2021). A graphical method for the preliminary design of ternary simple distillation columns at finite reflux. South African Journal of Chemical Engineering, 37, 99-109.
[11] Taifan G. S. P., Maravelias Ch. T. (2020). Integration of graphical approaches into optimization-based design of multistage liquid extraction. Computers \& Chemical Engineering, 143 (5), 107126.

[12] Yeoh K. P., Hui Ch. W. (2021). Rigorous NLP distillation models for simultaneous optimization to reduce utility and capital costs. Cleaner Engineering and Technology, 2, 100066

[13] Kister, R. (1990). Distillation Design, McGraw-Hill.

[14] Wehe A. H., Coates J. (1955). Vapor-liquid equilibrium relations predicted by thermodynamic examinations of activity coefficients. AIChE J., 1 (2), 241-246.

[15] Michalski H., Michalowski S., Serwinski M., Strumillo C. (1961). Vapour - Liquid Equilibria for the System Acetone - nButanol. Zesz. Nauk. Politech. Lodz. Chem., 10 (36), 73-84.

[16] Milton R., Chandler E., Brown S. F. (2021). Analysing the robustness of multi-stage bioseparations to measurement errors. Computer Aided Chemical Engineering, 50, 393398.

[17] Haan A. B., Eral H. B., Schuur B. (2020), Chapter 2. Evaporation and Distillation. Industrial Separation Processes: Fundamentals, Berlin, Boston: De Gruyter, 17-56.

[18] Morgan D. L. (2007), Use of transformed correlations to help screen and populate properties within databanks, Fluid Phase Equilib., 256, 54-61.

[19] Ghosh S., Seethamraju S. (2020). Reactive Distillation for Methanol Synthesis: Parametric Studies and Optimization Using a Non-polar Solvent. Process Integr Optim Sustain, 4, 325-342.

[20] Mahsa K., Abdoli S. M., (2021). The Design and Optimization of Extractive Distillation for Separating the Acetone/nHeptane Binary Azeotrope Mixture. ACS Omega, 6 (34), 22447-22453.

[21] Wankat, P. C. (2007). Separation Process Engineering, 2nd Ed., Prentice Hall.

[22] Frank O. (1977). Shortcuts for Distillation Design. Chem. Ing. March, 14, 110-124.

[23] Van Winkle M., Todd W. G. (1971), Optimum Fractionation Design by Simple Graphical Methods, Chem. Eng., 136.

[24] Fenske M. R. (1932). Fractionation of Straight-Run Pennsylvania Gasoline. Ind. Eng. Chem., 24 (5), 482-485.

[25] Underwood A. J. V. (1948). Fractional Distillation of Multicomponent Mixtures. Chem. Eng. Proc., 44 (8), 603-614.

[26] Kirkbridge C. G. (1944). Process Design Procedure for. Multicomponent Fractionators. Pet. Ref., 23 (9), 321-336.

[27] Renon H., Prausnitz J. M. (1968). Local Compositions in Thermodynamic Excess Functions for Liquid Mixtures, AIChE J., 14 (1), 135-144.

[28] Maurer G., Prausnitz J. M. (1978). Fluid Phase Equilibria, 2 (2), 91-99. 